\title{
Hysteresis Compensation of 3D Printed Sensors Using a Power Law Model for Various Input Signals
}

\author{
Martijn Schouten, Dimitrios Kosmas, Gijs Krijnen \\ Robotics And Mechatronics group, University of Twente, Enschede, The Netherlands \\ Email: m.schouten@utwente.nl
}

\begin{abstract}
We calculated the stability criterion for the modified power law model and subsequently adjust the model such that it's stability can be guaranteed. We applied both a sinusoid, a chirp, an exponentially decaying sine and bandwidth limited noise position excitation to a 3D printed symmetric piezoresistive cantilever and measure the differential response. A modified power law model is fitted directly to the inverse of the sensor data, to directly obtain a compensator for the sensor. The result was a stable compensator that reduced the hysteresis of the 3D printed sensor.
\end{abstract}

Keywords - 3D-Printing, Hysteresis, Compensation, Flexible, Soft, Tactile sensor, Power Law, Non-linear, Stability, Recurrent, Shallow, Neural Network

\section{INTRODUCTION}

3D printing is a promising technique for the fabrication of sensors, since the technique allows sensors to be produced cost effectively in low volumes, at high integration levels and considerable complexity [1], [2]. The technique is also well suited for the fabrication of soft sensors [3], [4].

A common material used in 3D printing of soft sensors is TPU, which is known to show visco-elastic behaviour [5]. Furthermore the piezoresistive elements that are often used, show a non-linear relationship and hysteresis [6]. Together both effects often result in hysteretic sensing behaviour.

To model hysteretic behaviour a circuit model was proposed by Biggio et al [7]. A simplified version of this model was shown to be able to significantly reduce the hysteresis in a 3D printed cantilever beam for a sinusoidal input [8]. In this paper the stability of this model is further improved and it is shown that this model can also be used to reduce the hysteresis for more complicated excitation signals.

\section{MODEL}

The model that is used is based on the model described in [7]. The model has an electrical circuit representation which is shown in figure 1 . The model consists of multiple cells consisting of a non-linear resistor and a capacitor (with $\mathrm{C}=1 \mathrm{~F}$ ). The voltage on the capacitors in subcircuit (a) is linked to the voltage of the voltage controlled voltage sources (VCCS's) in sub-circuit (b). These VCCS's, a VCCS directly linked to the

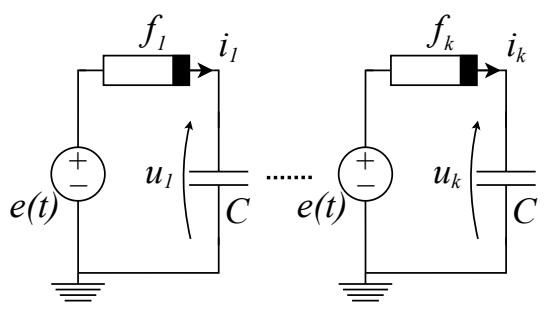

sub-circuit (a)

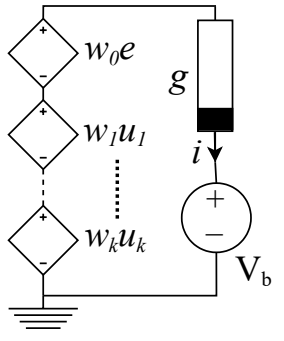

sub-circuit (b)
Fig. 1. A circuit representation of the model. Image courtesy of [7]

input and a fixed voltage source determine the voltage over a second non-linear resistor. The current through this second non-linear resistor is the output of the model. This circuit can be represented using a non-linear state space equation [8]:

$$
\begin{aligned}
\dot{u}_{k} & =f\left(e-u_{k}\right) \\
i & =g\left(w_{0} e+\sum_{k=1}^{N} w_{k} u_{k}-V_{b}\right)
\end{aligned}
$$

where $e$ is the input signal, $i$ is the output, $u_{k}$ are the voltages on the capacitors and $f$ and $g$ are non-linear functions. $w_{k}$ and $V_{b}$ are weights of the model and are to be fitted together with the parameters of the non-linear functions.

\section{A. Linearisation}

This non-linear state space equation can be linearised, around a certain state $u_{k, 0}$ and input $e_{0}$, by using a first order Taylor series approximation of the non-linear functions.

$$
\begin{aligned}
\dot{u}_{k} & =f_{k, 0}+f_{k, 0}^{\prime} e-f_{k, 0}^{\prime} u_{k} \\
i & =g_{0}+g_{0}^{\prime} w_{0} e+g_{0}^{\prime} \sum_{k=1}^{N} w_{k} u_{k}-g_{0}^{\prime} V_{b}
\end{aligned}
$$

With the following definitions:

$$
\begin{aligned}
& f_{k, 0}=f_{k}\left(u_{k, 0}-e_{0}\right), \quad g_{0}=g\left(w_{0} e_{0}+\sum_{k=1}^{N} w_{k} u_{k, 0}-V_{b}\right) \\
& f_{k, 0}^{\prime}=f_{k}^{\prime}\left(u_{k, 0}-e_{0}\right), \quad g_{0}^{\prime}=g^{\prime}\left(w_{0} e_{0}+\sum_{k=1}^{N} w_{k} u_{k, 0}-V_{b}\right)
\end{aligned}
$$




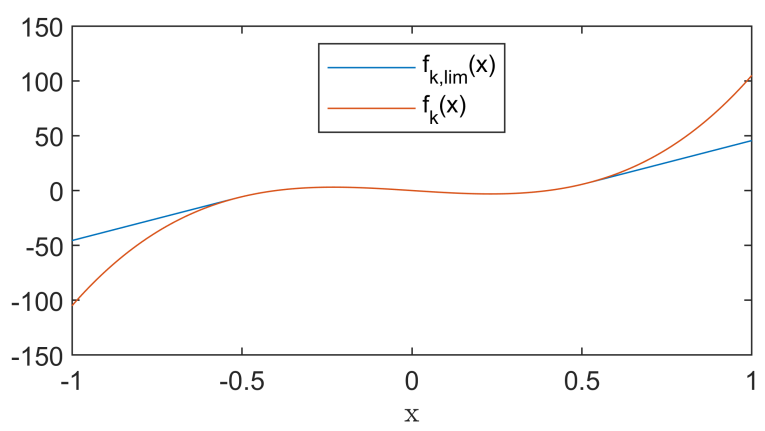

Fig. 2. Graphical illustration of the limit applied by equation 9

This can be written as a linear state space representation.

$$
\begin{aligned}
\dot{x} & =A x+B e \\
i & =C x+D e
\end{aligned}
$$

With:

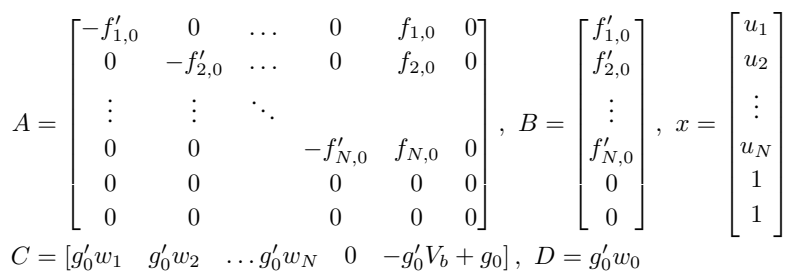

\section{B. Discretization}

This continuous state space equation can be discretized into the form:

$$
\begin{aligned}
x(k+1) & =A_{d} x(k)+B_{d} e(k) \\
i & =C_{d} x(k)+D_{d} e(k)
\end{aligned}
$$

When each matrix exponential is approximated using only the first two terms, only the $A$ matrix will change during discretization [9, p. 315]. This A matrix will change in the following way

$$
A_{d}=I+A \Delta t
$$

\section{Stability}

This system is asymptotically stable in case the magnitude of all eigenvalues of $A_{d}$ are less than unity [9, p. 329]. The eigenvalues of $A_{d}$ are located at $\lambda_{k}=1-f_{k, 0}^{\prime} \Delta t$ and at zero. This means the system is asymptotically stable in case:

$$
0<f_{k, 0}^{\prime}<\frac{2}{\Delta t}
$$

To prevent instability the function $f_{k}$ is limited such that:

$$
f_{k, \lim }= \begin{cases}f_{k}\left(x^{+}\right)+\frac{2}{\Delta t}\left(x-x^{+}\right) & \text {for } x>x^{+} \\ f_{k}(x) & \text { for } x^{-}>x>x_{+} \\ f_{k}\left(x^{-}\right)+\frac{2}{\Delta t}\left(x-x^{+}\right) & \text {for } x<x^{-}\end{cases}
$$

where $x^{-}$and $x^{+}$are respectively the minimum and the maximum value of the set of solutions of the equation $f^{\prime}(x)=$ $\frac{2}{\Delta t}$. Figure 2 graphically shows the implication of this limit. Note that this limitation allows the model to be unstable for $x^{-}>x>x_{+}$, but that such an instability always will cause the model to enter a part of the function where it is stable again, since the model is stable for both $x \rightarrow \infty$ and $x \rightarrow-\infty$.

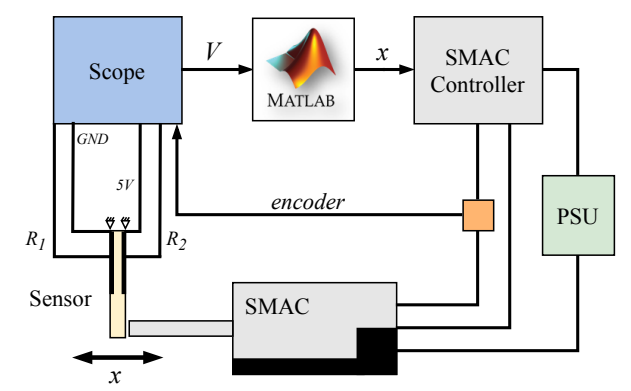

Fig. 3. Graphical illustration of the used measurements setup [8] (C)[2020] IEEE).

\section{MEASUREMENTS}

The used sensor and setup were previously described in [8] and [10]. In summary a linear actuator (SMAC LCA25-050$15 \mathrm{~F}$ ) is used to deform a $3 \mathrm{D}$ printed cantilever and a differential measurement of the resistance of piezoresistive elements on both sides of the cantilever is used to measure this deformation, see figure 3. An optical encoder is used as reference of the applied position signal. In this work two coupled Handyscope HS5 scopes sampling at $6.25 \mathrm{MHz}$ were used to measure the encoder signal and the voltage of two half bridges both with a $560 \Omega$ resistor, one of the piezoresistive elements and a DC supply voltage of $1 \mathrm{~V}$. A high sampling frequency was used in order to be able to have enough bandwidth to measure all the pulses of the $5 \mu \mathrm{m}$ resolution encoder.

Each measurement was executed twice in order to obtain one data set to fit the model to and one to validate the model on. A 2nd order zero-phase $20 \mathrm{~Hz}$ Butterworth filter was applied to the measured voltages and the decomposed encoder signal in order to reduce the noise in the measurement. After applying this filter all signals are downsampled to $40 \mathrm{~Hz}$ to speed up the model execution.

The difference between the relative resistance changes of both piezoresistors is calculated from the measured halfbridge voltages and used as the sensor output. The mean of the data was subtracted from both the sensor output and the encoder signal, to reduce the influence of drift that occurred in between the measurements. To normalise the data, the fitting and validation data are divided by the maximum of the absolute value of the fitting signals. The used excitation signals were:

1) A $0.5 \mathrm{~Hz}$ sinusoid of $200 \mathrm{~s}$ duration with an amplitude of $3 \mathrm{~mm}$.

2) A Chirp signal with a frequency exponentially increasing over a period of $100 \mathrm{~s}$ from $0.1 \mathrm{~Hz}$ to $3 \mathrm{~Hz}$ and an amplitude of $3 \mathrm{~mm}$

3) A sinusoid with a frequency of $0.5 \mathrm{~Hz}$ and an amplitude that exponentially decreases over $200 \mathrm{~s}$ from $2 \mathrm{~mm}$ to $100 \mu \mathrm{m}$.

4) Gaussian noise filtered with a $500^{\text {th }}$ order FIR lowpass filter with a $-3 \mathrm{~dB}$ cut-off frequency of $4 \mathrm{~Hz}$

In the rest of this paper these signals will be abbreviated as Sine, Chirp, Decay and Noise respectively. The sensor output is used as input for the model and the parameters of the model are optimised such that the model output predicts the position of the encoder. In this way the model can be used directly as a compensator for the hysteresis and non-linearity of the data. 
TABLE I. LEFT: INITIAL CONDITIONS OF THE USED POLYNOMIAL FUNCTIONS. RIGHT: ORDINARY COEFFICIENTS OF DETERMINATION

\begin{tabular}{c|cccccc|cc} 
& 0 & 1 & 2 & 3 & & & Model & Linear \\
\cline { 3 - 5 } & -1 & 0.1 & 0.1 & 1 & & Sine & 0.990 & 0.928 \\
$g(x)$ & 0.1 & 1 & 0.1 & 1 & & Chirp & 0.984 & 0.945 \\
& & & & & & Decay & 0.987 & 0.710 \\
Noise & 0.958 & 0.882
\end{tabular}

TABLE II. FITTED WEIGHTS

\begin{tabular}{c|cccc} 
& Sine & Chirp & Decay & Noise \\
\hline$f_{0}$ & -2.516 & -0.469 & -0.258 & -4.719 \\
$f_{1}$ & -2.666 & -1.712 & 0.663 & 3.412 \\
$f_{2}$ & 1.975 & -2.228 & -0.002 & -1.244 \\
$f_{3}$ & 1.953 & 3.125 & 0.008 & 1.469 \\
$g_{0}$ & 0.100 & -0.369 & -0.779 & -0.119 \\
$g_{1}$ & 1.195 & 1.094 & 1.320 & 1.125 \\
$g_{2}$ & 0.006 & 0.006 & -0.002 & 0.006 \\
$g_{3}$ & 0.475 & -0.384 & -0.240 & -0.259 \\
$w_{0}$ & 1.000 & 1.516 & 0.984 & 1.156 \\
$w_{1}$ & -0.492 & -0.531 & -0.875 & -0.406 \\
$V_{b}$ & 0.135 & -0.537 & -0.302 & 0.010
\end{tabular}

The fit is done using MATLAB's patternsearch running on all 6 cores of an Intel 179850 for $60 \mathrm{~s}$. Executing the model with the sine data takes $18 \mathrm{~ms}$. For both $f(x)$ and $g(x)$ a $3^{\text {th }}$ order polynomial of the following form is used:

$$
f(x)=f_{0}+f_{1} x+f_{2}^{2} x^{2}+f_{3}^{3} x^{3}
$$

The initial conditions of $f$ and $g$ can be found in table I on the left. In all cases $N=1$ and the initial condition of the remaining weights $w_{0}, w_{1}$ and $V_{b}$ were $1,-1$ and 0.01 respectively. As a reference of the performance, the model is compared to a linear fit, using MATLAB's polyfit.

\section{RESUlTS}

Figure 4 shows the hysteresis in the measured signals. Figure 5 shows the hysteresis of the model and the linear fit after they are applied to the data. The ordinary coefficient of determination between the encoder position and the position predicted by the the model as well as a linear fit can be found in table I on the right.

The fitted model parameters can be found in table II. The shape of the corresponding polynomials $f_{\text {lim }}(x)$ and $g(x)$ can be found in figure 6 . Without the limits set in equation 9 the fitted model of the chirp is unstable with the used validation data-set.

\section{DISCUSSION AND CONCLUSIONS}

A stability criterion for a modified power law model was derived. The model was subsequently adjusted to meet this criterion and it was shown that the model reduces the hysteresis of a 3D printed sensor in comparison to a linear fit, for several input signals. The used model only has 11 parameters and although the training was computationally intensive, the execution of the model was not. This research only shows a reduction of the hysteresis for the same sensor under the same conditions as was used during training. Further research is needed to determine the applicability in other situations.

Although a differential measurement is employed, which is expected to remove drift common to both strain gauges, it was necessary for a good fit that the mean of the signal was subtracted from both the fitting and the validation signals. Since the model itself does not remove inaccuracies due to drift further research on reducing drift is required when the sensors should be used for absolute force measurements.
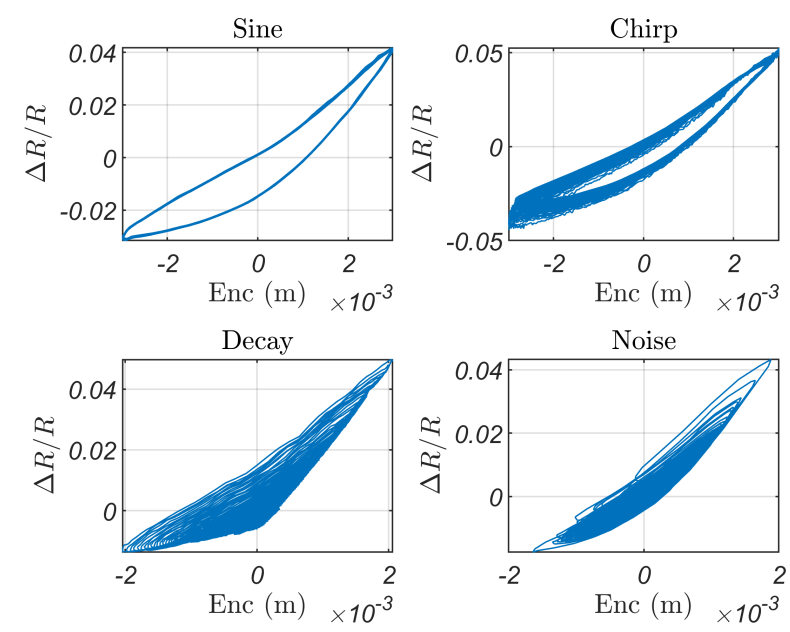

Fig. 4. Difference between the relative change in resistance of the two piezoresistive elements $(\Delta R / R)$ against the position measured by the encoder (Enc) for different excitation signals
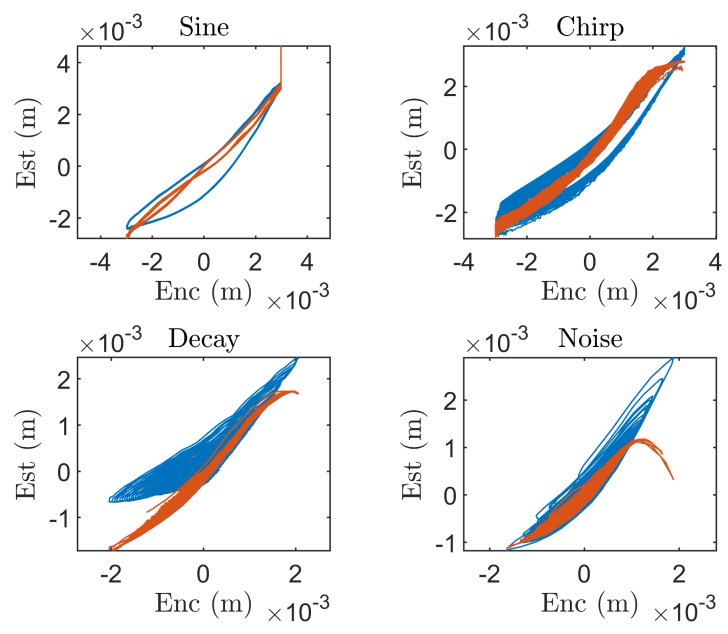

Fig. 5. Position estimated by the model (red) and a linear fit (blue) versus the position measured by the encoder (Enc) for different excitation signals
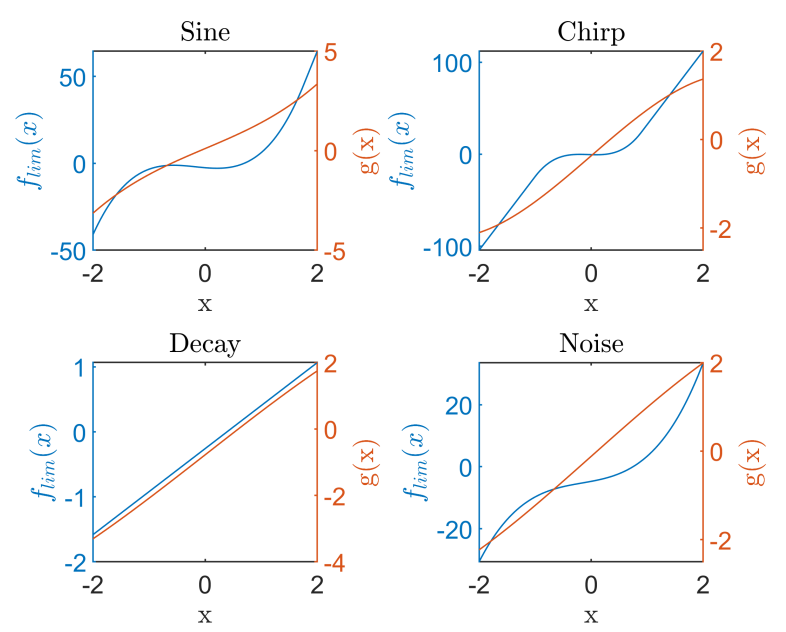

Fig. 6. The shape of the fitted $f_{\text {lim }}$ and $g$ functions 


\section{REFERENCES}

[1] T. Han, S. Kundu, A. Nag, and Y. Xu, "3D printed sensors for biomedical applications: A review," Sensors (Switzerland), vol. 19, no. 7, 2019.

[2] A. Dijkshoorn, P. Werkman, M. Welleweerd, G. Wolterink, B. Eijking, J. Delamare, R. Sanders, and G. J. Krijnen, "Embedded sensing: Integrating sensors in 3-D printed structures," Journal of Sensors and Sensor Systems, vol. 7, no. 1, pp. 169-181, 2018.

[3] G. Wolterink, R. Sanders, and G. Krijnen, "A flexible, three material, 3D-printed, shear force sensor for use on finger tips," Proceedings of IEEE Sensors, vol. 2019-Octob, pp. 2-5, 2019.

[4] M. O. F. Emon, F. Alkadi, D. G. Philip, D. H. Kim, K. C. Lee, and J. W. Choi, "Multi-material 3D printing of a soft pressure sensor," Additive Manufacturing, vol. 28, no. May, pp. 629-638, 2019. [Online]. Available: https://doi.org/10.1016/j.addma.2019.06.001

[5] H. J. Qi and M. C. Boyce, "Stress-strain behavior of thermoplastic polyurethanes," Mechanics of Materials, vol. 37, no. 8, pp. 817-839, 2005.

[6] J. Shintake, E. Piskarev, S. H. Jeong, and D. Floreano, "Ultrastretchable Strain Sensors Using Carbon Black-Filled Elastomer Composites and Comparison of Capacitive Versus Resistive Sensors," Advanced Materials Technologies, vol. 3, no. 3, pp. 1-8, 2018.

[7] M. Biggio, A. Oliveri, F. Stellino, M. Parodi, and M. Storace, "A circuit model of hysteresis and creep," IEEE Transactions on Circuits and Systems II: Express Briefs, vol. 62, no. 5, pp. 501-505, 2015.

[8] D. Kosmas, M. Schouten, and G. Krijnen, "Hysteresis Compensation of 3D Printed Sensors by a Power Law Model with Reduced Parameters," FLEPS 2020 - IEEE International Conference on Flexible and Printable Sensors and Systems, Proceedings, 2020.

[9] K. Ogata, Discrete-Time Control Systems (2nd Ed.). USA: PrenticeHall, Inc., 1995.

[10] M. Schouten, B. Prakken, R. Sanders, and G. Krijnen, "Linearisation of a $3 \mathrm{~d}$ printed flexible tactile sensor based on piezoresistive sensing," in 2019 IEEE SENSORS, Oct 2019, pp. 1-4. 